\title{
Potencial antihiperglicémico de Gentianella gilgiana (Reimers) Fabris ex J.S. Pringle en la absorción intestinal de glucosa in vitro en ratas albinas diabéticas
}

Ana María del Carmen Guevara-Vásquez* 1

RESUMEN

Objetivo: Evaluar el potencial antihiperglicémico de la infusión de Gentianella gilgiana (Reimers) Fabris ex J.S. Pringle en la absorción intestinal de glucosa in vitro en Rattus norvegicus cepa Holtzman con diabetes inducida químicamente. Materiales y métodos: Estudio de tipo experimental y analítico. Se realizó el tamizaje fitoquímico de la planta. Para el estudio in vitro se utilizaron veinte ratas albinas machos, a las que se les indujo diabetes utilizando aloxano monohidratado (Sigma-Aldrich, Saint Louis, MO, EE. UU.) en dosis de $120 \mathrm{mg} / \mathrm{kg}$ p.c, vía intraperitoneal, y luego fueron distribuidas al azar en dos grupos de diez ratas albinas cada uno. Luego de diez días fueron sacrificados para extraerles un segmento intestinal con la técnica del saco intestinal evertido, y medir el transporte y absorción de glucosa. En el grupo control (intestinos evertidos incubados en solución Krebs-Henseleit glucosado) y en el grupo experimental (intestinos incubados en solución Krebs Henseleit glucosado más la infusión de Gentianella gilgiana al 10\% p/v), se tomaron muestras basales de los líquidos de incubación, luego otras muestras a los 15, 30, 45 y 60 minutos para cuantificar la glucosa de los compartimentos mucoso y seroso mediante el método enzimático de la glucosa oxidasa.

Resultados: Se evidenciaron menores concentraciones de glucosa absorbida en compartimento seroso intestinal del grupo experimental (G. gilgiana) con diferencia estadísticamente significativa respecto al control $(p<0,05)$.

Conclusiones: La infusión de Gentianella gilgiana (Reimers) Fabris ex J.S. Pringle presenta potencial antihiperglicémico significativo que disminuye la absorción intestinal de glucosa in vitro en ratas albinas diabéticas.

Palabras clave: Hiperglicemia; Gentianella; Absorción intestinal; Diabetes mellitus (Fuente: DeCS BIREME).

\section{Antihyperglycemic potential of Gentianella gilgiana (Reimers) Fabris ex J.S. Pringle for in vitro intestinal glucose absorption in diabetic albino rats}

\section{ABSTRACT}

Objective: To evaluate the antihyperglycemic potential of the Gentianella gilgiana (Reimers) Fabris ex J.S. Pringle infusion for in vitro intestinal glucose absorption in Rattus norvegicus of the Holtzman strain with chemically-induced diabetes.

Materials and methods: The study had an experimental and analytical design. A phytochemical screening of the whole plant was carried out. For the in vitro study, twenty male albino rats were induced with diabetes using alloxan monohydrate (Sigma-Aldrich, Saint Louis, MO, USA) at a dose of $120 \mathrm{mg} / \mathrm{kg}$ bw administered by intraperitoneal route, and then randomly distributed into two groups of ten albino rats each. After ten days, they were sacrificed to extract an intestinal segment using the everted intestinal sac technique, and measure the glucose transport and absorption. In the control group (everted intestines incubated in Krebs-Henseleit solution containing glucose) and the experimental group (intestines incubated in Krebs-Henseleit solution containing glucose plus the Gentianella gilgiana (Reimers) Fabris ex J.S. Pringle infusion at $10 \% \mathrm{w} / \mathrm{v}$ ), basal samples of fluids were taken from incubation, and then other samples were taken at 15, 30, 45 and 60 minutes to quantify the glucose from the mucous and serous compartments using the glucose oxidase enzymatic method.

Results: Lower concentrations of absorbed glucose were observed in the intestinal serous compartment of the experimental group (G. gilgiana) with a statistically significant difference compared to the control group ( $\mathrm{p}<0.05)$. Conclusions: The Gentianella gilgiana (Reimers) Fabris ex J.S. Pringle infusion exhibits a significant antihyperglycemic potential by decreasing the in vitro intestinal glucose absorption in diabetic albino rats.

Keywords: Hyperglycemia; Gentianella; Intestinal absorption; Diabetes mellitus (Source: MeSH NLM).

1. Universidad Nacional de Trujillo, Facultad de Farmacia y Bioquímica. Trujillo, Perú.

*Autor corresponsal 


\section{INTRODUCCIÓN}

La diabetes mellitus (DM) es una enfermedad crónica y compleja que requiere atención médica continua con estrategias de reducción de riesgos multifactoriales, más allá del control glucémico (1). En el Perú, en el año 2017, un reporte del Instituto Nacional de Estadística e Informática del Perú (INEI) menciona que el 3,3\% de la población de 15 y más años de edad informó que fue diagnosticada con diabetes mellitus. Las mujeres fueron las más afectadas $(3,6 \%)$, en relación a los hombres $(3,0 \%)$. Asimismo, el mayor porcentaje de personas con diabetes se encuentran en Lima Metropolitana $(4,1 \%)$ y resto de la costa $(4,0 \%)$, y menor porcentaje en la sierra $(1,8 \%)$ y selva $(2,7 \%)^{(2)}$.

A nivel mundial, aproximadamente, la mitad de las muertes atribuibles a la hiperglucemia ocurren antes de los 70 años. Según proyecciones de la OMS, la diabetes será la séptima causa de mortalidad en $2030^{(3)}$.

La característica fisiopatológica es la hiperglicemia, además de poliuria, polidipsia, polifagia y puede ser secundaria a cualquier enfermedad que cause destrucción extensa de los islotes pancreáticos, como pancreatitis, tumores, ciertos fármacos, algunas endocrinopatías genéticas o adquiridas y escisión quirúrgica ${ }^{(4,5)}$. La glucosa es el azúcar principal de la sangre que sirve a los tejidos como el principal combustible metabólico, y la glicemia depende, entre otros factores, del aporte de carbohidratos de la dieta, los cuales deben ser digeridos y la glucosa resultante absorbida. Los factores que intervienen en la regulación de la glicemia y que pueden evidenciar alteraciones cuando fallen o se encuentren afectados son la absorción intestinal, utilización tisular, producción endógena de glucosa, factores hormonales y excreción renal ${ }^{(5)}$.

Debido a que la glicemia postprandial comienza con la digestión de los carbohidratos, que para ingresar al intestino primero son transformados en glucosa, luego son transportados a través del borde en cepillo de la mucosa intestinal mediante transporte activo secundario (cotransportador $\mathrm{Na}^{+}$-glucosa, SGLT1), y de allí, pasan a la sangre mediante difusión facilitada. Actualmente, se están estudiando los mecanismos de disminución de la glucosa postprandial, sobre todo, el de drogas de origen vegetal que reducen la absorción intestinal de la glucosa (6-8).

En la medicina tradicional existen diversas plantas empleadas en el tratamiento de la diabetes, entre ellas Momordica charantia, Bauhinia magalandra, Taraxacum officinale ${ }^{(7)}$, Gentianella thyrsoidea ${ }^{(9)}$, G. umbellata ${ }^{(10)}$, entre otras. La especie Gentianella gilgiana (Reimers)
Fabris ex J.S. Pringle, de nombre común "yangamarsha"o "genciana", es originaria del Perú, pertenece a la familia Gentianaceae, género Gentianella, es una hierba perenne que crece en la jalca del departamento de La Libertad y su hábitat natural son las laderas rocosas a una altura de 4200 a 4600 m s. n. m. (11-13) . Esta planta es utilizada entera en forma de infusión por los pobladores de Huamachuco, como medicina folklórica en el tratamiento de la diabetes.

Se tiene poca información científica sobre esta especie en particular, lo que motivó el interés de realizar este trabajo de investigación para proporcionar más información acerca del potencial antidiabético de esta especie en particular. La finalidad de esta investigación fue determinar el potencial antihiperglicémico de Gentianella gilgiana (Reimers) Fabris ex J.S. Pringle, preparada como una infusión, en la absorción intestinal de glucosa in vitro en Rattus norvegicus cepa Holtzman con diabetes inducida químicamente por aloxano.

\section{MATERIALES Y MÉTODOS}

\section{Diseño y población de estudio}

Se realizó un estudio de tipo experimental y analítico. Se utilizaron 20 Rattus norvegicus cepa Holtzman, machos, desparasitados, aparentemente sanos, adultos de 10 semanas de edad, y con un peso corporal entre 185 a 220 g. Fueron adquiridos en el Instituto Nacional de Salud-Chorrillos, Lima; luego alojados en el Bioterio de la Facultad de Farmacia y Bioquímica de la Universidad Nacional de Trujillo durante 15 días previos al estudio, para la aclimatación respectiva; fueron alimentados con dieta estándar y agua ad libitum, y mantenidos a una temperatura ambiental entre 22 y $24{ }^{\circ} \mathrm{C}$, humedad relativa del $70 \%$ y con ciclo de luz/oscuridad de 12/12 horas.

Se utilizó la planta completa de Gentianella gilgiana (Reimers) Fabris ex J.S. Pringle, yangamarsha (Figura 1), recolectada en los alrededores del cerro Huaylillas (altitud: $4200 \mathrm{~m} \mathrm{~s}$. n. m., entre coordenadas geográficas latitud sur 749'36" y longitud oeste $78^{\circ} 02^{\prime} 46^{\prime \prime}$ ) en el distrito de Huamachuco, provincia de Sánchez Carrión, departamento de La Libertad. La identificación de la especie vegetal fue realizada por un especialista biólogo y curador botánico del Herbarium Truxillense (HUT) de la Universidad Nacional de Trujillo; el Herbario es una Unidad académica de la Facultad de Ciencias Biológicas. Se utilizaron $2 \mathrm{~kg}$ de la planta completa, se eliminaron las sustancias extrañas, se lavó con agua destilada y se llevó a secado en estufa con aire circulante a $40{ }^{\circ} \mathrm{C}$ durante 3 días. La muestra fue pulverizada y tamizada hasta obtener partículas de $2 \mathrm{~mm}$ de diámetro. 


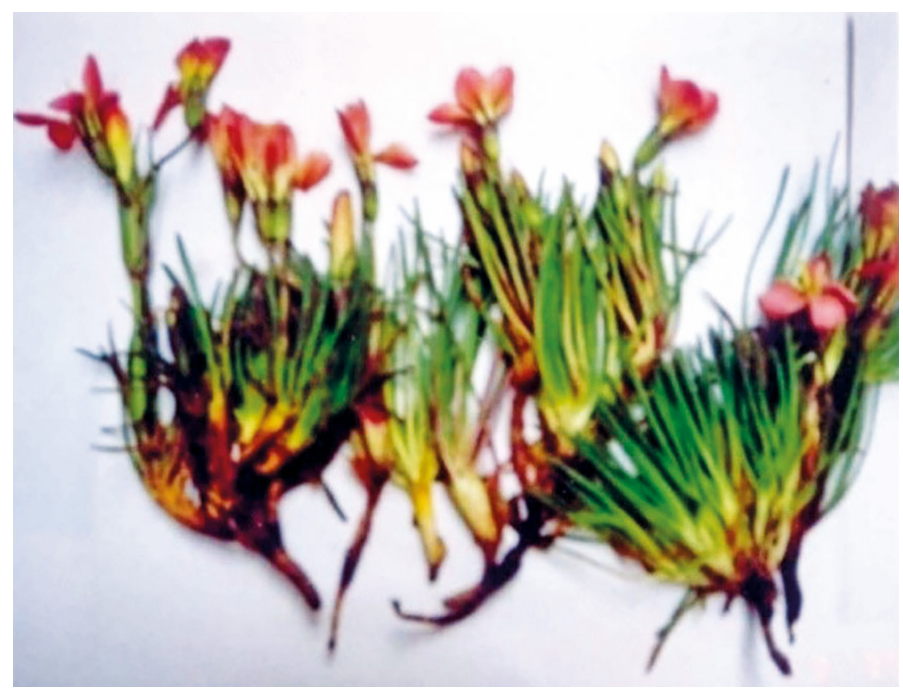

Figura 1. Especie vegetal Gentianella gilgiana (Reimers) Fabris ex J.S Pringle, (yangamarsha)

Variables y mediciones

Administración de la infusión de Gentianella gilgiana (Reimers) Fabris ex J.S. Pringle. Efectos en la absorción intestinal de glucosa.

Tamizaje fitoquímico y preparación de la infusión al 10 \%

El tamizaje fitoquímico de Gentianella gilgiana (Reimers) Fabris ex J.S. Pringle se realizó según la metodología de Lock ${ }^{(14)}$. Se pesó un gramo de residuo seco, se agregó $30 \mathrm{~mL}$ del disolvente (según sea el caso), para obtener los extractos diclorometano, ácido etanólico, ácido acuoso y acuoso. Se mantuvo a reflujo controlado durante 10 minutos en baño de agua, se enfrió y filtró con papel Whatman $\mathrm{N}^{\circ} 1$. Los análisis de identificación química, de coloración, precipitación , o ambas, fueron realizados para determinar la presencia de fitoconstituyentes: flavonoides (prueba de Shinoda), alcaloides (pruebas de Dragendorff, Hager, Mayer y Wagner), compuestos fenólicos (prueba del cloruro férrico), saponinas (prueba de la espuma), taninos (prueba de la gelatina), antraquinonas y naftoquinonas (prueba de Bornträger), esteroides y triterpenos (prueba de Liebermann-Burchard), leucoantocianidinas (prueba de Rosenheim).

La infusión de Gentianella gilgiana (Reimers) Fabris ex J.S. Pringle al $10 \%(\mathrm{p} / \mathrm{v})$, extracto acuoso, fue preparada momentos antes de ser utilizada. Se pesaron $10 \mathrm{~g}$ de muestra pulverizada y tamizada, luego se vertió sobre ella $100 \mathrm{~mL}$ de agua hirviendo, se dejó en contacto por cinco minutos y luego se procedió a filtrar (15) para dejar entibiar. El filtrado se evaporó al vacío para dar un residuo (rendimiento: 12,6 \%). El extracto seco se almacenó bajo refrigeración a $4{ }^{\circ} \mathrm{C}$ en una botella estéril de color ámbar.

Inducción de diabetes

Doce horas previas a la inducción, se privó de alimentos a las ratas albinas. Luego se les administró vía intraperitoneal $120 \mathrm{mg} / \mathrm{kg}$ p.c, de aloxano monohidratado sol. $3 \%$, preparado con solución salina isotónica y mantenido muy frío previo a la inyección (16). Una hora después, las ratas albinas tuvieron acceso libre de alimento y agua. A las 24 horas de administrado el aloxano se determinaron los valores de glucosa en sangre utilizando el kit para determinación de glucosa enzimática (Laboratorios Wiener). El mantenimiento de la hiperglicemia ( $298 \mathrm{mg} / \mathrm{dL}$ en promedio), después de diez días de la inyección de aloxano, se tomó como índice del daño pancreático y establecimiento del estado diabético.

Los animales de experimentación diabéticos fueron divididos al azar en dos grupos de diez especímenes cada uno (control y experimental). El grupo control estuvo en ayunas por 16 horas y con suministro de agua ad libitum, antes de aplicar la eversión del saco intestinal para evaluar la absorción intestinal de glucosa. En el grupo experimental se siguió el mismo procedimiento que para el grupo control, pero se adicionó la dosis de $3,7 \mathrm{mg} / \mathrm{mL}$ de la infusión al $10 \%(\mathrm{p} / \mathrm{v})$ de Gentianella gilgiana (Reimers) al medio de incubación en el equipo de órgano aislado. 


\section{Eversión de los sacos intestinales}

A los diez días de la inducción de diabetes, se sacrificaron las ratas albinas en ayuno de 14 horas, se abrió el abdomen y se ubicó el duodeno, luego se cortó cerca de la unión duodeno-yeyunal y se obtuvieron segmentos de unos $15 \mathrm{~cm}$ de largo de yeyuno. Se lavó con solución salina fisiológica fría. Para la eversión del intestino, se siguió la técnica descrita por Wilson y Wiseman (17) y reproducida por otros investigadores (18-20), que fue modificada para este estudio, reemplazando el material de la varilla, que era de acero inoxidable, por polietileno, con aumento ligero de la longitud (Figura 2). Inmediatamente después de extraer la pieza de intestino y everterla con una varilla de polietileno $(1,5 \mathrm{~mm}, 350 \mathrm{~mm})$, se colocó en una placa petri con solución salina, adecuada oxigenación y temperatura a $37{ }^{\circ} \mathrm{C}$. Se ligó firmemente un extremo del segmento intestinal con una pieza de hilo y se pesó, luego el saco vacío se llenó con 1,5 mL de solución de
Krebs sin glucosa con una jeringa sin aguja insertada en el extremo aún sin ligar y después de llenado se ligó este extremo del segmento y se llevó a pesar nuevamente. La pieza de intestino fue colocada en el equipo de órgano aislado conteniendo $100 \mathrm{~mL}$ de solución Krebs-Henseleit buffer glucosada, controlado a $37{ }^{\circ} \mathrm{C}$ de temperatura, con agitación de 110 vibraciones/min y oxigenación constante mediante burbujeo con una mezcla gaseosa de $95 \%$ de $0_{2}$ y $5 \%$ de $\mathrm{CO}_{2}$ durante la incubación. $\mathrm{El} \mathrm{pH}$ fue mantenido a 7,4. La solución de Krebs-Henseleit buffer bicarbonato fue preparada con los siguientes componentes: cloruro de sodio $118 \mathrm{mM} / \mathrm{L}$, cloruro de potasio $4,7 \mathrm{mM} / \mathrm{L}$, cloruro de calcio 1,2 $\mathrm{mM} / \mathrm{L}$, bicarbonato de sodio $25 \mathrm{mM} / \mathrm{L}$, sulfato de magnesio $1,2 \mathrm{mM} / \mathrm{L}$, fosfato de sodio monobásico 1,2 $\mathrm{mM} / \mathrm{L}$, EDTA disódico $9,7 \mathrm{mg} / \mathrm{L}$, agua destilada c. $\mathrm{s}$. $\mathrm{p}$. $1000 \mathrm{~mL}, \mathrm{pH} 7,4$. La glucosa (2,00 g/L) se agregó al medio de incubación antes de empezar a trabajar los intestinos evertidos) ${ }^{(18,19)}$.

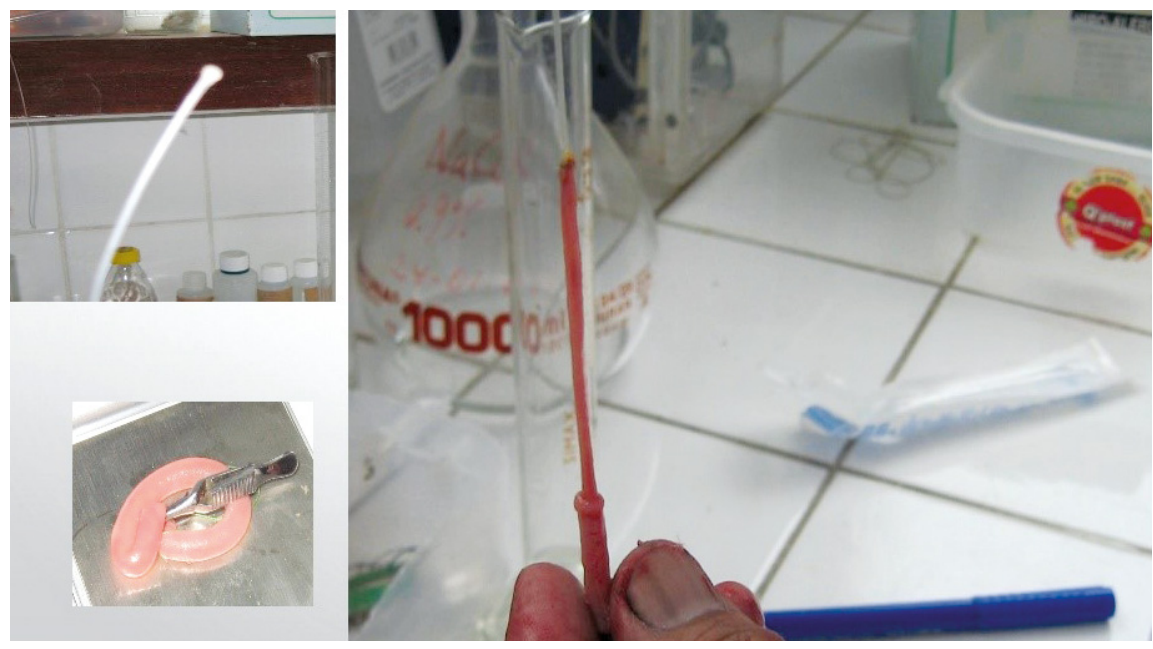

Figura 2. Eversión de intestino delgado de Rattus norvegicus cepa Holtzman

Cuantificación de la glucosa en lado mucoso intestinal (medio de incubación KHB glucosado)

En los grupos control y experimental se tomaron muestras basales del medio de incubación (solución buffer de KrebsHenseleit glucosada) para cuantificar la glucosa (mg/dL) con el kit enzimático del laboratorio Wiener. Luego se tomaron otras muestras del medio de incubación a los 15 , 30, 45 y 60 minutos. Específicamente, en caso del grupo experimental, se adicionó al medio de incubación, la infusión de Gentianella gilgiana (Reimers) a dosis 3,7 mg/ $\mathrm{mL}$ y se realizó el mismo procedimiento que para el grupo control.
Cuantificación de la glucosa absorbida a nivel intestinal (lado seroso)

En ambos grupos, al final de la incubación, los sacos intestinales fueron retirados del baño de órgano y pesados nuevamente. Para determinar la glucosa absorbida in vitro en el saco intestinal de rata albina, el líquido contenido en el lado seroso fue recolectado en un tubo de ensayo, tras realizar una pequeña incisión en el saco, y llevado también a la cuantificación de glucosa $(\mathrm{mg} / \mathrm{dL})$, con el kit enzimático del laboratorio Wiener; luego los sacos intestinales ya vacíos de cada grupo fueron pesados nuevamente. Los pesos de los sacos vacíos antes y después de la incubación no variaron de manera significativa. 


\section{Análisis estadístico}

Los datos obtenidos fueron procesados y analizados con pruebas estadísticas, que tomaron como parámetro central la media aritmética, y como medida de dispersión se determinó la desviación estándar. El estudio comparativo se realizó mediante el análisis de varianza (ANOVA) de dos vías seguido de prueba HSD de Tukey y la prueba $\mathrm{T}$ de Student para muestras independientes. Los valores de $p<0,05$ se consideraron como estadísticamente significativos. Para analizar los datos se usó el paquete estadístico SPSS V. 22.

\section{Consideraciones éticas}

Todos los procedimientos de este estudio fueron realizados acorde con las pautas de la Guía para el cuidado y el uso de animales de laboratorio ${ }^{(21)}$ y los reglamentos establecidos por el Comité de Ética para la Investigación con animales de la Universidad Nacional de Trujillo (Res. Cons. Univ. N $0247-2016 /$ UNT). La investigación fue aprobada por el Comité de Ética de la Facultad de Farmacia y Bioquímica de la Universidad Nacional de Trujillo.

\section{RESULTADOS}

\section{Tamizaje fitoquímico}

En el cribado fitoquímico de la planta completa de $G$. gilgiana (Reimers) Fabris ex J.S. Pringle se encontró presencia de esteroides, cumarinas, leucoantocianidinas, triterpenos, alcaloides, flavonoides y taninos (Tabla1). Se evidenció mayor presencia de flavonoides y taninos en el extracto acuoso.

Tabla 1. Tamizaje fitoquímico de Gentianella gilgiana (Reimers) Fabris ex J.S. Pringle

\begin{tabular}{|c|c|c|c|c|c|}
\hline \multirow[b]{2}{*}{$\begin{array}{l}\text { Metabolito } \\
\text { secundario }\end{array}$} & \multirow[b]{2}{*}{$\begin{array}{l}\text { Nombre de la } \\
\text { prueba }\end{array}$} & \multicolumn{4}{|c|}{ Extractos de Gentianella gilgiana (Reimers) Fabris ex J.S. Pringle } \\
\hline & & DM & $E$ & AA & A \\
\hline \multirow[t]{2}{*}{ Alcaloides } & Mayer & - & + & + & ++ \\
\hline & Dragendorff & - & + & + & ++ \\
\hline Flavonoides & Shinoda & - & ++ & + & ++ \\
\hline Esteroides & Liebermann-Burchard & + & + & + & + \\
\hline Quinonas & Bornträger & - & - & - & + \\
\hline Fenoles & FeCL3 & - & + & + & + \\
\hline Taninos & Gelatina & - & ++ & + & ++ \\
\hline Leucoantocianidinas & Rosenheim & + & + & + & ++ \\
\hline Saponinas & Espuma & - & - & - & - \\
\hline Cumarinas & $\mathrm{NaOH} 10 \%$ & + & ++ & - & + \\
\hline
\end{tabular}

DM: extracto diclometánico, E: extracto etanólico, AA: extracto acuoso-ácido, A: extracto acuoso. Presencia e intensidad: (-): ausencia; $(+)$ : poco; $(++)$ : moderado; $(+++)$ : abundante

Potencial antihiperglicémico in vitro de G. gilgiana (Reimers) Fabris ex J.S. Pringle (G.G)

Concentraciones de glucosa en compartimento mucoso intestinal

En la figura 3 se evidencia el efecto que tiene la infusión de G.G al $10 \%$ en la concentración de glucosa del medio de incubación, solución KHB glucosada, que está en contacto directo con el compartimento mucoso del saco intestinal evertido de la rata albina diabética.
En el caso del grupo control, es notoria la disminución de glucosa en el medio de incubación a partir de los 30 minutos y se hace más evidente al final de los 60 minutos ( $99 \mathrm{mg} / \mathrm{dL}$ ), a diferencia del grupo experimental (con la infusión de G.G) con $159 \mathrm{mg} / \mathrm{dL}$ de glucosa en medio de incubación a los 60 minutos, lo que evidencia un menor transporte y absorción de glucosa hacia el saco intestinal en el grupo experimental $(p<0,01)$, a diferencia del grupo control, en el cual la glucosa evidencia mayor disminución de su concentración en el medio KHB. 


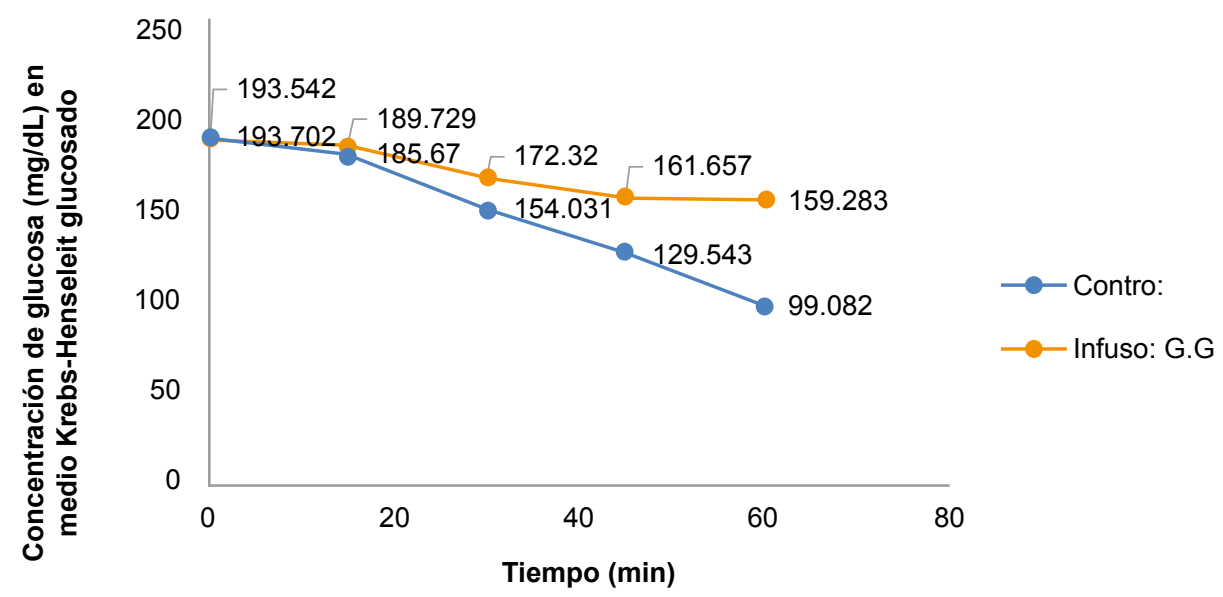

Figura 3. Promedios de las concentraciones de glucosa $(\mathrm{mg} / \mathrm{dL})$ en compartimento mucoso intestinal de Rattus norvegicus cepa Holtzman diabéticos

Incubación realizada en solución Krebs Henseleit glucosado (control) y en solución Krebs Henseleit glucosado más la infusión de Gentianella gilgiana 10 \% (G.G.). Los valores en cada grupo $(n=10)$ se expresan como la media \pm D.S.M. (ANOVA de dos vías/ post hoc HSD de Tukey); ${ }^{*} p<0,05 ;{ }^{* *} p<0,01$ : expresa diferencia estadísticamente significativa.

Absorción intestinal de glucosa en el lado seroso del saco intestinal evertido

La tabla 2 muestra las concentraciones promedio de glucosa absorbida in vitro en el compartimento seroso del saco intestinal evertido de ratas albinas diabéticas. El aumento de glucosa en el líquido seroso fue indicativo de la glucosa transportada desde el medio de incubación KHB glucosado en el equipo de órgano aislado. Al finalizar los sesenta minutos de incubación, en el grupo control, la absorción de glucosa en el lado seroso intestinal, se produce en un $41 \%$; a diferencia del grupo experimental, en el que la infusión de G.G del medio de incubación permite que la glucosa solo se absorba en un $15 \%$ hacia el lado seroso intestinal, lo que evidencia una diferencia estadísticamente significativa $(p<0,01)$.

Tabla 2. Promedios de concentración de glucosa absorbida in vitro en lado seroso de saco intestinal evertido a los sesenta minutos de incubación

\begin{tabular}{|c|c|c|c|}
\hline \multicolumn{4}{|c|}{ Glucosa absorbida in vitro en lado seroso del saco intestinal } \\
\hline & \multicolumn{3}{|c|}{ Concentración de glucosa (mg/dL) } \\
\hline & \multirow{3}{*}{$\begin{array}{c}\text { Condición inicial: } \\
\text { basal 0, } \\
(\mathrm{mg} / \mathrm{dL})\end{array}$} & \multicolumn{2}{|c|}{ A los 60 minutos } \\
\hline \multirow[b]{2}{*}{ Grupo $(n=10)$} & & Compartimento seroso & Compartimento mucoso \\
\hline & & $\begin{array}{l}\text { Solución saco intestinal } \\
\text { evertido: Absorción de } \\
\text { glucosa }\end{array}$ & $\begin{array}{l}\text { En solución Krebs } \\
\text { Henseleit glucosado }\end{array}$ \\
\hline \multirow[b]{2}{*}{ Control } & \multicolumn{2}{|r|}{$78,56 \pm 5,38$} & $99,08 \pm 6,21$ \\
\hline & $193,70 \pm 3,34$ & $\%^{\mathrm{A}} \quad 40,55$ & $\%$ B 51,15 \\
\hline \multirow{2}{*}{$\begin{array}{l}\text { Gentianella gilgiana (Reimers) } \\
\text { Fabris ex J.S. Pringle al } 10 \% \text { ) }\end{array}$} & \multirow{2}{*}{$193,54 \pm 3,32$} & $29,13 \pm 1,48^{* *}$ & $159,28 \pm 2,81$ \\
\hline & & $\% A 15,05$ & $\%$ B 82,29 \\
\hline
\end{tabular}

\%A: porcentaje de absorción de glucosa en el saco intestinal; \%: porcentaje de glucosa que queda en el frasco de incubación después de una hora. Los valores en cada grupo $(n=10)$ se expresan como la media \pm D.S.M. Prueba T de Student para muestras independientes. ${ }^{* *} p<0,01$ : expresa diferencia estadísticamente significativa respecto al control. 
Potencial antihiperglicémico de Gentianella gilgiana (Reimers) Fabris ex J.S. Pringle en la absorción intestinal de glucosa in vitro en ratas albinas diabéticas

\section{DISCUSIÓN}

Uno de los parámetros más importantes a tener en cuenta en el tratamiento y control de la diabetes mellitus, así como un factor de riesgo para las enfermedades cardiovasculares, es la hiperglicemia postprandial (22). Los procesos de absorción de glucosa en el intestino delgado se producen en dos etapas. Primero, la glucosa es transportada desde el lumen intestinal hacia el interior del enterocito por el trasportador SGLT-1 y después, cuando se acumula dentro de la célula, se moviliza a favor de su gradiente de concentración a través de la célula hasta la membrana basolateral y luego, por difusión facilitada (GLUT-2), llega al plasma ${ }^{(7,23)}$, por lo que, posterior a la ingesta de carbohidratos (principalmente glucosa), en la etapa postprandial los niveles de glucosa aumentan.

Los resultados obtenidos en esta investigación muestran que en el grupo control de las ratas albinas diabéticas, la concentración de glucosa en el medio de incubación KHB (lado mucoso) va disminuyendo de manera significativa a medida que transcurre el tiempo hasta el final del período de incubación, en contraste a lo que sucede por efecto de la infusión de $G$. gilgiana $(\mathrm{p}<0,01)$. Esto se explicaría por la absorción aumentada de glucosa en el intestino del animal diabético, ya que estudios evidencian que, en el estado de diabetes, se encuentra aumentada la absorción intestinal de glucosa debido al incremento de los trasportadores de glucosa SGLT-1 y GLUT-2 en el enterocito ${ }^{(24,25)}$.

La diabetes inducida en ratas albinas utilizando aloxano, se produce por destrucción parcial de las células beta pancreáticas mediante reacciones de óxido-reducción en las que se forman radicales libres que afectan directamente la producción de insulina ${ }^{(16)}$, y es, en este estado de diabetes, en que se evidencia la mayor absorción intestinal de glucosa en el grupo control.

La infusión de Gentianella gilgiana (Reimers) Fabris ex J.S. (G.G) ejerce una acción notoria en el transporte y absorción intestinal de glucosa desde la solución Krebs Henseleit glucosada (compartimento mucoso) hacia el saco intestinal evertido de la rata albina (compartimento seroso), en donde se evidencia menor absorción de glucosa comparada al grupo control, ya que la concentración de glucosa en el medio de incubación no disminuye en la misma proporción en que lo hace en el grupo control; lo que pudiera deberse a la presencia de fitoconstituyentes como flavonoides, leucoantocianinas, taninos, esteroides, cumarinas, alcaloides, entre otros metabolitos secundarios encontrados en el tamizaje fitoquímico de G.G.
En los últimos años se ha dado gran importancia al estudio del efecto inhibitorio en la absorción intestinal in vitro de glucosa con el uso de plantas con potencial antidiabético, se encontró, por un lado, una modificación en la permeabilidad de la membrana de los enterocitos, lo que disminuyó la absorción intestinal de glucosa en saco intestinal evertido de ratas, se atribuyen estos efectos a los alcaloides, leucoantocianinas, taninos y fenoles contenidos como compuestos activos del extracto crudo de hojas de Erytroxylum macrocarpum (18), compuestos que también posee nuestra planta en estudio. Por otro lado, también se reportó bloqueo de los cotransportadores de $\mathrm{Na}^{+}$- glucosa que se encuentran en la membrana apical de los enterocitos, como en los estudios de los flavonoides de plantas medicinales como Bauhinia megalandra ${ }^{(7)}$. Asimismo, se ha reportado la inhibición del transporte de glucosa en la membrana del borde en cepillo intestinal, por la alteración del gradiente $\mathrm{Na}^{+}-\mathrm{K}^{+}$ en la membrana del enterocito, efectos producidos por los extractos de Momordica charantia y Tea sinensis ${ }^{(26,27)}$.

La menor absorción de glucosa hacia el lado seroso de los intestinos evertidos de ratas albinas diabéticas, evidenciada en nuestra investigación, se debería, probablemente, al efecto que ejercerían los metabolitos secundarios o fitoconstituyentes de la infusión de Gentianella gilgiana (Reimers), los cuales inhibirían - bloquearían el transporte de glucosa a través del intestino delgado al inhibir al cotransportador $\mathrm{Na}+-$ glucosa, el SGLT-1, tal como se reportó en el caso de las especies Gymnema sylvestre y Zizyphus jujuba, cuyos flavonoides, leucoantocianinas, polifenoles, isoflavonas, xantonas, taninos, saponinas y otros compuestos demostraron tener potente efecto inhibitorio del SGLT1 y actividad antihiperglicémica ${ }^{(28)}$. Asimismo, estudios reportan que Malus domestica Borkh y Allium cepa L. evidenciaron inhibición de SLGT-1 y de GLUT-2, efecto que se atribuye a los flavonoides de dichas plantas ${ }^{(8,29)}$. Asimismo, los compuestos fenólicos del té, además de inhibir a las glucosidasas también inhibieron a SGLT-1 (27).

Otros mecanismos de acción de los metabolitos secundarios que se han reportado serían el retraso en la transferencia de glucosa del estómago al intestino delgado, lugar principal para la absorción; otros pueden inhibir enzimas digestivas, $a$-amilasas y a-glucosidasas, y también inhibir el transporte activo de glucosa a través de la membrana de borde en cepillo de los enterocitos (19,30).

En base a lo analizado, la menor absorción intestinal de glucosa en los sacos intestinales evertidos de las ratas albinas, que se evidencia en el grupo experimental (infusión de Gentianella gilgiana (Reimers) Fabris ex J.S) 
podría deberse a la inhibición del transportador SGLT$1 \mathrm{y}$, posiblemente, del transportador GLUT-2. Por otro lado, la disminución del ingreso de glucosa al intestino delgado podría deberse a la inhibición de la bomba extractora $\mathrm{Na}+-\mathrm{K}+-$ ATPasa en el enterocito, por parte de los fitoconstituyentes de G.gilgiana, por lo que se pierde el gradiente necesario para que la glucosa ingrese a la célula mediante transporte activo secundario. Estos efectos se deberían a la presencia de flavonoides, leucoantocianinas, alcaloides, taninos y otros compuestos bioactivos encontrados en esta planta.

En conclusión, la infusión de Gentianella gilgiana (Reimers) Fabris ex J.S. Pringle presenta potencial antihiperglicémico al disminuir significativamente la absorción intestinal de glucosa in vitro en Rattus norvegicus cepa Holtzman con diabetes inducida por aloxano.

\section{REFERENCIAS BIBLIOGRÁFICAS}

1. American Diabetes Association. Standards of medical care in diabetes-2019. Diabetes Care 2019; 42(Suppl. 1): S1-S2.

2. Instituto Nacional de Estadística e Informática. Perú, 2017. Enfermedades no transmisibles y transmisibles [Internet]. Lima, 2018. Disponible en: https://www.inei.gob.pe/media/ MenuRecursivo/publicaciones_digitales/Est/Lib1526/libro. pdf

3. Mathers C, Loncar D. Projections of global mortality and burden of disease from 2002 to 2030. PLoS Med. 2006; 3(11): e442.

4. Porter R, Kaplan J. El Manual Merck. $19^{\circ}$ ed. Buenos Aires: Médica Panamericana; 2014.

5. Stryer L, Berg J, Tymoczko J. Bioquímica con aplicaciones clínicas. $7^{\circ}$ ed. Madrid: Reverté. S.A; 2013.

6. Estudante M, Morais J, Soveral G, Bennet L. Intestinal drug transporters: An overview. Advanced Drug Delivery Reviews. 2013; 65(10): 1340-56.

7. Márquez A, Motta N, González-Mujica F. Efectos de extractos vegetales sobre la absorción intestinal de glucosa y su captación por vesículas de membrana apical de enterocitos. Revista de la Facultad de Medicina. Universidad Central de Venezuela; 2002.

8. Schulze C, Bangert A, Kottra G, Geillinger K, Schwanck B, Vollert $\mathrm{H}$; et al. Inhibition of the intestinal sodium-coupled glucose transporter 1 (SGLT1) by extracts and polyphenols from apple reduces postprandial blood glucose levels in mice and humans. Mol, Nutr. Food Res. 2014; 58: 1795-1808.

9. Gutiérrez M. Fitoconstituyentes de Gentianella thyrsoidea y su efecto hipoglicemiante en Oryctolagus cunniculus. [Tesis].

10. Salazar J. Contribución al estudio químico y farmacológico de Gentianella umbellata (G. Don) Fabris. [Tesis]. Lima: Pontificia Universidad Católica del Perú. Facultad de Ciencias; 2003.

11. López A. Catálogo de la flora del Departamento de La Libertad Arnaldoa, 1998; 5(2): 215-263.

12. The International Plant Names Index (IPNI). Plant names details. 2015. Disponible en: http://www.ipni.org/ipni/ idPlantNameSearch. do?id=943420-1

13. Rybcznski J, Davey M, Mikula A. The Gentianaceae-Volume 1: Characterization and ecology. Heidelberg: Springer; 2014.

14. Lock O. Investigación fitoquímica. $2^{\circ}$ ed. Lima: Edit. Pontificia Universidad Católica del Perú.; 1994.

15. Gennaro A. Remington Farmacia $21^{\circ}$ ed. Madrid: Panamericana S.A; 2012.

16. Verma L, Khatri A, Kaushik B, Patil U, Pawar R. Antidiabetic activity of Cassia occidentalis (Linn) in normal and alloxaninduced diabetic rats. Indian J Pharmacol. 2010; 42(4): 224-8.

17. Wilson T, Wiseman $G$. The use of sacs of everted small intestine for the study of the transference of substances from the mucosal to the serosal surface. J Physiol. 1954; 123: 11625.

18. Mahomoodally M, Fakim A, Subratty A. Effects of Erythoxylum macrocarpum, an endemic medicinal plant of Mauritius, on the transport of monosaccharide, amino acid and fluid across rat everted intestinal sacs in vitro. J Cell Mol Biol 2005; 4: 93-8.

19. Hamilton K, Butt G. Glucose transport into everted sacs of the small intestine of mice. Adv Physiol Educ. 2013; 37: 15-426.

20. Pingili R, Vemulapilli S, Mullapudi S, Nuthakki S, Pendyala $\mathrm{S}$, Kilaru N. Pharmacokinetic interaction study between flavanones (hesperetin, naringenin) and rasagiline mesylate in wistar rats. Drug Develop Ind Pharm. 2016; 42(7): 1110-7.

21. National Research Council (NRC). Academy of Sciences. Guide for the care and use of laboratory animals. $8^{\circ} \mathrm{ed}$. Washington: The National Academies Press; 2011.

22. Ceriello A. Postprandial hyperglycemia and diabetes complications is it time to treat? Diabetes. 2005; 54: 1-7.

23. Yusoff N, Ahmad M, Al-Hindini B, Widyawati T, Yam M, Mahmud $\mathrm{R}$, et al. Aqueous Extract of Nypa fruticans Wurmb. Vinegar Alleviates Postprandial Hyperglycemia in Normoglycemic Rats. Nutrients. 2015; 7, 7012-26.

24. Jara E, Hidalgo J, Flores: C Pérez M, Yáñez A, Hidalgo A, et al. Estudio de un extracto estandarizado de Maqui rico en delfininas en el mantenimiento del balance de glucosa. Rev Farmacol Chile. 2012; 5(2): 27-34.

25. Kellet G, Helliwell P. The diffusive component of intestinal glucose absorption is mediated by the glucose-induced recruitment of GLUT-2 to the brush-border membrane. Biochem J. 2000; 350: 155-62.

26. Mahomoodally $M$, Subratty A. A kinetic model for in vitro intestinal uptake of L-tyrosine and $D(+)$ glucose across rat everted gut sacs in the presence of Momordica charantia, a medicinal plant used in traditional medicine against diabetes mellitus. J Cell Mol Biol. 2004; 3: 39-44.

27. Kreydiyyeh, S. Abdel-Hasan B, Churukian Z. Tea extracts inhibits intestinal absorption of glucose and sodium in rats. Comp Biochem Physiol C Pharmacol Toxicol Endocrinol. 2003; 117(4): 5-78.

28. Ashok K, Madhusudana R. Diabetes mellitus and multiple therapeutic approaches of phytochemicals: Present status and future prospects. Curr Sci. 2002; 83(1): 30-6.

29. Blaschek W. Natural product as lead compounds for sodium glucose cotransporter (SGLT) inhibitors. Planta Med. 2017; 83(12/13): 985-993. 
Potencial antihiperglicémico de Gentianella gilgiana (Reimers) Fabris ex J.S. Pringle en la absorción intestinal de glucosa in vitro en ratas albinas diabéticas

30. Barthe L, Woodley J, Hovin G. Gastrointestinal absorption of drugs: methods and studies. Fundamental and Clinical Pharmacology. 1999; 13(2): 154-68.

Fuentes de financiamiento:

Este artículo ha sido financiado por la autora.

Conflictos de interés:

La autora declara no tener ningún conflicto de interés.

Correspondencia:

Ana María del Carmen Guevara Vásquez

Dirección: Av. Juan Pablo Il s/n- Ciudad Universitaria. Trujillo, Perú. Teléfono: $(+51)$ 44-474839

Correo electrónico: aguevara@unitru.edu.pe

\section{Recibido: 18 de junio de 2019. \\ Evaluado: 28 de junio de 2019 \\ Aprobado: 08 de julio de 2019.}

๑ La revista. Publicado por Universidad de San Martín de Porres, Perú. (cc) Br Licencia de Creative Commons Artículo en acceso abierto bajo términos de Licencia Creative Commons Atribución 4.0 Internacional. (http://creativecommons.org/licenses/by/4.0/)

ORCID iDs

Ana María del Carmen Guevara Vásquez (iD) https: / /orcid.org/0000-0002-1320-5959 